\title{
BMJ Open Associations between hyponatraemia, volume depletion and the risk of falls in US hospitalised patients: a case- control study
}

\author{
Elizabeth A Fehlberg, ${ }^{1,2,3}$ Robert J Lucero, ${ }^{1,2,4}$ Michael T Weaver, ${ }^{1}$ \\ Anna M McDaniel, ${ }^{1}$ A Michelle Chandler, ${ }^{5}$ Phyllis A Richey, ${ }^{6}$ Lorraine C Mion, ${ }^{7}$ \\ Ronald I Shorr $2,8,9$
}

To cite: Fehlberg EA, Lucero RJ, Weaver MT, et al. Associations between hyponatraemia, volume depletion and the risk of falls in US hospitalised patients: a case-control study. BMJ Open 2017;7:e017045. doi:10.1136/ bmjopen-2017-017045

- Prepublication history for this paper is available online. To view these files please visit the journal online (http://dx.doi org/10.1136/bmjopen-2017017045).

Received 27 March 2017 Revised 29 May 2017 Accepted 21 June 2017

CrossMark

For numbered affiliations see end of article.

Correspondence to

Elizabeth A Fehlberg;

efehlberg@rti.org

\section{ABSTRACT}

Objective We aimed to determine if abnormal laboratory values which may indicate volume depletion are associated with increased odds of experiencing a hospitalacquired fall.

Design Matched case-control study.

Setting Four hospitals located in the Southeast USA. Participants Data from 699 adult fallers and 1189 matched controls (non-fallers) were collected via chart review from 2005 to 2010 . Controls were matched to cases by nursing unit, time of fall and length of stay.

Outcome measures The primary exposures included serum sodium, blood urea nitrogen (BUN), creatinine, BUN/creatinine ratio and haematocrit. Conditional logistic regression with $\mathrm{m}: \mathrm{n}$ matching was used to determine adjusted and unadjusted ORs.

Results Serum sodium levels were strongly associated with falls. In models controlling for demographic and other fall risk factors, patients with serum sodium levels of $125 \mathrm{mEq} / \mathrm{L}$ or less were associated with increased odds of experiencing a fall as compared with those with serum sodium levels of greater than $134 \mathrm{mEq} / \mathrm{L}$ (adjusted OR $(\mathrm{aOR})=5.08,95 \% \mathrm{Cl}$ 1.43 to 18.08). Conversely, elevated BUN, creatinine and elevated BUN/creatinine ratios were not associated with increased odds of experiencing a fall $(\mathrm{aOR}=0.64,95 \% \mathrm{Cl} 0.49$ to $0.84 ; \mathrm{aOR}=0.70,95 \% \mathrm{Cl} 0.54$ to 0.92 and $\mathrm{aOR}=0.77,95 \%$ Cl 0.58 to 1.04, respectively.)

Conclusions Laboratory indices that may indicate volume depletion appear to be unrelated to falls. However, hyponatraemia does appear to be a risk factor for falls, and those with serum sodium levels below $126 \mathrm{mEq} / \mathrm{L}$ are at especially high risk. It may be that other deficits associated with hyponatraemia, like altered mental status, are associated with risk of experiencing a hospital-acquired fall. These results indicate that abnormal laboratory values, like low sodium, can be useful for identifying hospitalised patients at risk of falling. Therefore, further investigation into abnormal laboratory values as predictors of hospital-acquired falls is warranted.

\section{INTRODUCTION}

Each year, between 700000 and 1000000 falls occur in US hospitals with associated total direct medical costs of $\$ 34$ billion. ${ }^{12}$
Strengths and limitations of this study

- The main strengths of this study include: (1) a large sample size collected over 6 years, (2) the setting which included four hospitals and (3) a matching strategy that controls for environmental and staffing factors at the time of a fall event.

- Only laboratory values that are not specific for volume depletion were used as potential indicators of volume depletion instead of other indicators such as urine output or orthostatic blood pressure.

- Our design did not account for all environmental factors like location of patient room relative to the nursing station.

- Due to the nature of observational research, we cannot exclude the possibility of incomplete control of potential confounders and there was a small number of patients with serum sodium levels of $125 \mathrm{mEq} / \mathrm{L}$ or less.

- The generalisability of our findings is limited by the context of our study and the sampling frame which relied on only patients whose laboratory results were known 24 hours prior to the fall index time.

In addition, falls are the leading cause of both fataland non-fatalinjuriesamongolderadults. ${ }^{1}$ Numerous studies have been conducted to examine the associations between patientlevel risk factors and hospital-acquired falls. Examples of previously identified risk factors include dementia, diabetes, depression, cognitive status and impaired mobility. ${ }^{3-9}$ Although many researchers have examined demographic factors and health conditions, few studies have aimed to understand the underlying biological mechanisms related to falling. Laboratory values, which have generally been neglected in previous falls research, can potentially increase our understanding of risk factors for hospital-acquired falls. ${ }^{10}$

Researchers have created tools to assist in the identification of patients at risk for falls 
including the Morse Fall Scale, the STRATIFY Scale and the Hendrich Fall Risk Model. ${ }^{411} 12$ These tools include risk factors such as history of falls, mobility impairments and altered elimination. However, the predictability of these tools has been shown to vary considerably when subjected to external validations. ${ }^{13}$ This indicates that there is a potential for improvement which could be achieved with the inclusion of additional factors like laboratory values. Researchers have examined the diagnosis of anaemia, haemoglobin levels and/or haematocrit levels as potential risk factors for hospital-acquired falls. ${ }^{3} 8$ 14-22 However, there has been limited examination of other laboratory values. Previous research has examined hyponatraemia in a psychiatric population, hyponatraemia and hypokalaemia in a Japanese cohort, albumin in a postoperative population, and creatinine, sodium and the ratio of blood urea nitrogen (BUN) to creatinine. ${ }^{1823-25}$ In addition, one study examined bivariate associations between approximately 30 laboratory values and falling. ${ }^{17}$ However, all of the aforementioned non-anaemia laboratory work was limited in sample size, with approximately 100 fallers.

Previous research has identified volume depletion as a risk factor for falling, but to the best of our knowledge there has been limited examination of volume depletion and hospital-acquired falls. We found one study that examined the relationship between fluid intake and falls in a nursing home and found a significant decline in falls $(p=0.05)$ during a hydration programme. ${ }^{26}$ However, further examination of this potential relationship is warranted, especially since nursing home and hospital populations differ. Abnormal serum creatinine, BUN, sodium and haematocrit levels can indicate multiple health conditions, including volume depletion. ${ }^{1027-35}$ Specifically, abnormal laboratory values which may indicate volume depletion include high BUN levels $(>21 \mathrm{mg} / \mathrm{dL})$, high creatinine levels $(>1.1 \mathrm{mg} / \mathrm{dL}$ in females and $>1.2 \mathrm{mg} / \mathrm{dL}$ in males), high BUN to creatinine ratios $(>20)$, high haematocrit levels ( $>45 \%$ in females and $>51 \%$ in males) or high and low sodium levels $(>145 \mathrm{mEq} / \mathrm{L}$ and $<135 \mathrm{mEq} / \mathrm{L}$, respectively). ${ }^{36} 37$ To address whether volume depletion is potentially related to hospital-acquired falls, we used a case-control design to test if abnormal BUN, creatinine, haematocrit and sodium levels were associated with odds of a hospital-acquired fall.

\section{METHODS}

\section{Conceptual framework}

This study is informed by Choi's conceptual multisystemic fall prevention model. ${ }^{38}$ Intrinsic risk factors, including physiological factors (ie, laboratory values), are directly associated with fall risk. In addition, symptoms secondary to physiological changes are associated with environmental and technological interventions. By discovering physiological intrinsic risk factors, modifications to the environment and care processes can theoretically lead to a reduction in falls.

\section{Study population}

This case-control study was conducted using data that were collected between 2005 and 2010 across four hospitals. These hospitals are located in the Southeastern USA and belong to the same health system. Data from the largest hospital, a 635-bed urban community facility, were collected from 15 medical-surgical units. Data from the three other suburban community hospitals, ranging in size from 200 to 260 beds, were collected from nine medical-surgical units. A case was defined as an adult patient who fell on one of the 24 study units during the study period as reported in the hospital incident reporting system. In this study, a fall was defined as an unintentional change in position which resulted in coming to rest on the ground or a lower level. Falls resulting from catastrophic clinical events (eg, seizure, stroke or arrhythmia) were excluded. For each case, we identified up to two controls with a similar hospital length of stay that were on the same nursing unit at the same time the case fell. The data collection was approved by the Institutional Review Board of the University of Tennessee Health Science Center. This secondary analysis was approved by the University of Florida Institutional Review Board.

\section{Exposures}

Exposures were determined for both cases and controls using medical records review by personnel blinded to the case status of the patient. We designated an index time for the controls based on the date and time that the matched case fell. This index time was used to establish the timeframe for collecting the exposures. Specifically, we recorded exposures most proximate to the time of the fall or the index time for controls. In addition, we excluded exposures that were greater than 24 hours before the fall event. Our primary exposures were serum sodium and other abnormal laboratory values that may indicate volume depletion including high BUN, creatinine, BUN to creatinine ratio and haematocrit. Volume depletion occurs when there is a decrease in circulating blood volume, which can occur as a result of inadequate fluid intake or excessive loss of fluids or blood. The abnormal laboratory value classifications can be found in table $2 .^{36}$

\section{Covariates}

Demographic covariates in this study included age, race and gender. We also adjusted for the medical conditions of Parkinson's disease, dementia, hypertension, congestive heart failure (CHF), diabetes mellitus and stroke. Presence of these comorbid conditions was defined as a positive history in the medical record. Also, we controlled for whether the patient had experienced an acute mental status change within the past 24 hours prior to the fall index time. In addition, we controlled for the patient's fall risk score prior to the fall index time. This score is based on the Morse Fall Scale with the inclusion of medications which potentially affect mobility or cognition (eg, sedatives, antipsychotics, antidepressants, diuretics and 
opiates).${ }^{11}$ We based the binary measure of high fall risk (yes/no) on this standardised tool which is used across the four hospitals.

\section{Statistical analysis}

Data analysis was performed using SAS software V.9.4 of the SAS System for Windows (SAS Institute, Cary, North Carolina, USA). Univariate descriptive statistics were calculated by fall status for the overall sample. Descriptive statistics including mean and SD were calculated for continuous data, and counts and percentages were calculated for categorical data. Potential multicollinearity issues were evaluated by testing the correlation between categorical exposures using the phi coefficient. Further, multicollinearity diagnostics were evaluated by checking condition index and variable inflation values. Since cases either had one or two controls, and were matched based on potential confounders, conditional logistic regression with m:n matching was used to determine bivariate and multivariable associations. ${ }^{39} 40$ Using this approach, the unadjusted and adjusted ORs were estimated along with their corresponding 95\% CIs. A series of multivariable models were created. Each multivariable model included one of the abnormal laboratory values of interest and the potential covariates. Potential covariates were included in the multivariable models regardless of bivariate $p$ values.

Additional analyses were performed on serum sodium levels to further analyse the potential relationship between hyponatraemia and hospital-acquired falls. A categorical variable was created which included three levels of serum sodium including $125 \mathrm{mEq} / \mathrm{L}$ or lower, 126 to $134 \mathrm{mEq} / \mathrm{L}$ and $135 \mathrm{mEq} / \mathrm{L}$ or greater. These categories were created to determine if there was a doseresponse association between hospital-acquired falls and sodium levels. Conditional logistic regression with m:n matching was used to determine the bivariate and multivariable associations. Using this approach, the unadjusted and adjusted ORs were estimated along with their corresponding 95\% CIs. Two models were created including (1) an unadjusted model which only included the categorical sodium level variable and (2) an adjusted model which included the categorical sodium level variable and the covariates of high BUN, high creatinine, high BUN to creatinine ratio, age, race, gender, high fall risk score, acute mental status change, and the medical conditions of dementia, hypertension, CHF, diabetes and stroke.

\section{RESULTS}

The final sample included 699 fallers and 1189 matched controls. Descriptive statistics by fall status for the final sample can be found in table 1 . Controls were aged 62 years on average and $59.7 \%$ female, whereas the cases were aged 65 years on average and $50.9 \%$ female. In addition, only $35.8 \%$ of controls compared with $55.7 \%$ of cases were assessed as high risk prior to the fall index time. Also, $75.4 \%$ of cases had a diagnosis of hypertension, whereas $68.5 \%$ of controls had a diagnosis of hypertension. We did not include the exposures of Parkinson's disease, high haematocrit and high sodium in further analyses due to low frequencies of less than $3 \%$ in this sample. Specifically, $1.72 \%$ of cases and $1.01 \%$ of controls had a diagnosis of Parkinson's disease, $1.45 \%$ of cases and $1.09 \%$ of controls had high haematocrit levels, and $2.11 \%$ of cases and $2.40 \%$ of controls had high sodium levels.

Unadjusted ORs from bivariate conditional logistic regression models can be found in table 2 . In the unadjusted model, the presence of low sodium increased the odds of a hospital-acquired fall ( $\mathrm{OR}=1.485,95 \%$ CI 1.136 to 1.940). In contrast, the presence of elevated BUN levels decreased the odds of a hospital-acquired fall $(\mathrm{OR}=0.785,95 \%$ CI 0.622 to 0.991$)$. The presence of elevated creatinine levels and BUN to creatinine ratios were not significantly associated with the occurrence of a hospital-acquired fall in the unadjusted models.

Adjusted ORs from the four multivariable conditional logistic regression models can also be found in table 2 . Each of these models included one abnormal laboratory value which may indicate volume depletion while controlling for the covariates of age, race, gender, high fall risk score, acute mental status change, and the medical conditions of dementia, hypertension, CHF, diabetes and stroke. After adjusting for these covariates, the presence of low sodium significantly increased the odds of a hospital-acquired fall by $36 \%(p=0.04)$. Presence of high BUN and high creatinine were significantly associated with decreased odds of experiencing a hospital-acquired fall $(\mathrm{OR}=0.643, \mathrm{p}=0.001$, and $\mathrm{OR}=0.700, \mathrm{p}=0.009$, respectively). However, a high BUN to creatinine ratio was not significantly associated with experiencing a hospital-acquired fall in the adjusted model. The results of these four models indicate that based on abnormal laboratory values, volume depletion may not be associated with increased odds of a hospital-acquired fall.

Categories of serum sodium levels including $125 \mathrm{mEq} / \mathrm{L}$ or lower, 126 to $134 \mathrm{mEq} / \mathrm{L}$ and $135 \mathrm{mEq} / \mathrm{L}$ or greater were analysed further. Adjusted and unadjusted ORs from conditional logistic regression models for these categories of serum sodium levels can be found in table 3. Two models were created including an unadjusted model and a model adjusted for presence of high BUN, high creatinine, a high BUN to creatinine ratio, age, race, gender, high fall risk score, acute mental status change, and the medical conditions of dementia, hypertension, CHF, diabetes and stroke. In the unadjusted model, those with a serum sodium level of $125 \mathrm{mEq} / \mathrm{L}$ or lower were 4.82 times more likely to experience a hospital-acquired fall as compared with a serum sodium level of $135 \mathrm{mEq} / \mathrm{L}$ or greater (95\% CI 1.54 to 15.12 ). In the adjusted model, those with a serum sodium level of $125 \mathrm{mEq} / \mathrm{L}$ or lower were 5.08 (95\% CI 1.43 to 18.08 ) times more likely to experience a hospital-acquired fall. In addition, figure 1 displays the proportion of cases (fallers) and controls (non-fallers) among the three categories of serum sodium levels. Of those with a serum sodium level of $125 \mathrm{mEq} / \mathrm{L}$ or lower, approximately $71 \%$ 
Table 1 Characteristics of cases and controls by fall status $(\mathrm{N}=1888)$

\begin{tabular}{|c|c|c|c|c|c|c|c|}
\hline \multirow[b]{2}{*}{ Factor } & \multicolumn{3}{|c|}{ Controls $(n=1189)$} & \multicolumn{4}{|c|}{ Cases $(n=699)$} \\
\hline & $\mathbf{n}$ & Frequency (\%) & Mean (SD) & $\mathbf{n}$ & Frequency (\%) & Mean (SD) & p Value* \\
\hline Age & 1189 & & $61.6(17.8)$ & 699 & & $64.9(16.1)$ & $<0.01$ \\
\hline Race & 1189 & & & 698 & & & $<0.01$ \\
\hline White & & $538(45.3)$ & & & $392(56.2)$ & & \\
\hline Not White & & $651(54.8)$ & & & $306(43.8)$ & & \\
\hline Gender & 1189 & & & 698 & & & $<0.01$ \\
\hline Male & & $479(40.3)$ & & & $343(49.1)$ & & \\
\hline Female & & $710(59.7)$ & & & $355(50.9)$ & & \\
\hline Hospital & 1187 & & & 699 & & & $0.979 \dagger$ \\
\hline University & & 528 (44.5) & & & $320(45.8)$ & & \\
\hline Community 1 & & $285(24.0)$ & & & $164(23.5)$ & & \\
\hline Community 2 & & $97(8.2)$ & & & $57(8.2)$ & & \\
\hline Community 3 & & 277 (23.3) & & & $158(22.6)$ & & \\
\hline High fall risk & 1169 & 419 (35.8) & & 684 & $381(55.7)$ & & $<0.01$ \\
\hline Mental status change & 1183 & 224 (18.9) & & 692 & $180(26.0)$ & & $<0.01$ \\
\hline \multicolumn{8}{|l|}{ Medical conditions } \\
\hline Parkinson's disease & 1185 & $12(1.0)$ & & 698 & $12(1.7)$ & & 0.14 \\
\hline Dementia & 1185 & $139(11.7)$ & & 698 & $106(15.2)$ & & 0.06 \\
\hline Hypertension & 1188 & $814(68.5)$ & & 699 & $527(75.4)$ & & $<0.01$ \\
\hline $\mathrm{CHF}$ & 1186 & 252 (21.3) & & 694 & $160(23.1)$ & & 0.35 \\
\hline Diabetes & 1186 & $394(33.2)$ & & 696 & $261(37.5)$ & & 0.07 \\
\hline Stroke & 1185 & $143(12.1)$ & & 695 & $108(15.5)$ & & 0.03 \\
\hline
\end{tabular}

${ }^{*} p$ Values were determined using conditional logistic regression with $\mathrm{m}: \mathrm{n}$ matching. †Cases and controls were matched by unit and therefore matched by hospital as well. $\mathrm{CHF}$, congestive heart failure.

were fallers and $29 \%$ were non-fallers. In contrast, of those with a serum sodium level of 126 to $134 \mathrm{mEq} / \mathrm{L}$ and $135 \mathrm{mEq} / \mathrm{L}$ or greater, approximately $48 \%$ and $38 \%$ were fallers and $52 \%$ and $62 \%$ were non-fallers, respectively.

\section{DISCUSSION}

In this study, we identified important associations between patient-level risk factors and hospital-acquired falls. Of the four variables chosen as indicators of volume depletion

Table 2 Bivariate and multivariable conditional logistic regression of abnormal BUN, creatinine and sodium values on hospital-acquired falls

\begin{tabular}{|c|c|c|c|c|c|}
\hline Model & Factor & Controls (n) & Cases (n) & Unadjusted OR (95\% Cl) & Adjusted OR* $(95 \% \mathrm{Cl})$ \\
\hline \multirow[t]{2}{*}{1} & $\mathrm{BUN}>21 \mathrm{mg} / \mathrm{dL}$ & 337 & 213 & 0.785 (0.622 to 0.991$)$ & $0.643(0.494$ to 0.838$)$ \\
\hline & $\mathrm{BUN} \leq 21 \mathrm{mg} / \mathrm{dL}$ & 579 & 411 & Reference & Reference \\
\hline 2 & $\mathrm{Cr} \leq 1.1 \mathrm{mg} / \mathrm{dL} \ddagger$ & 556 & 397 & Reference & Reference \\
\hline 3 & $\mathrm{BUN} / \mathrm{Cr}>20$ & 215 & 140 & 0.860 (0.657 to 1.124$)$ & 0.766 (0.578 to 1.042$)$ \\
\hline \multirow[t]{2}{*}{4} & $\mathrm{Na} \geq 135 \mathrm{mEq} / \mathrm{L}$ & 762 & 469 & Reference & Reference \\
\hline & $\mathrm{Na}<135 \mathrm{mEq} / \mathrm{L}$ & 155 & 148 & 1.485 (1.136 to 1.940$)$ & 1.356 (1.013 to 1.816$)$ \\
\hline
\end{tabular}

${ }^{*}$ Conditional logistic regression model adjusted for age, race, gender, high fall risk score, acute mental status change, and medical conditions of dementia, hypertension, congestive heart failure, diabetes and stroke.

†In males $>1.2 \mathrm{mg} / \mathrm{dL}$.

$\neq$ In males $\leq 1.2 \mathrm{mg} / \mathrm{dL}$.

BUN, blood urea nitrogen; $\mathrm{Cr}$, creatinine. 
Table 3 Bivariate and multivariable conditional logistic regression of serum sodium levels on hospital-acquired falls

\begin{tabular}{|c|c|c|c|c|}
\hline Serum sodium (mEq/L) & Controls (n) & Cases (n) & Unadjusted OR (95\% Cl) & $\begin{array}{l}\text { OR adjusted for multiple risk factors* } \\
(95 \% \mathrm{Cl})\end{array}$ \\
\hline 125 or lower & 5 & 12 & 4.82 (1.54 to 15.12$)$ & 5.08 (1.43 to 18.08$)$ \\
\hline 135 or greater & 762 & 469 & Reference & Reference \\
\hline
\end{tabular}

${ }^{*}$ Conditional logistic regression model adjusted for high BUN, high creatinine, high BUN to creatinine ratio, age, race, gender, high fall risk score, acute mental status change, and medical conditions of dementia, hypertension, congestive heart failure, diabetes and stroke. BUN, blood urea nitrogen.

that were usable in the analyses, only low sodium was independently related to increased odds for falling in the hospital setting. However, the abnormal laboratory values of high BUN levels and high creatinine levels were significantly associated with decreased odds of experiencing a hospital-acquired fall. It is possible that high BUN and creatinine levels appear to be protective because high BUN and creatinine levels can result in fatigue. ${ }^{36} \mathrm{~A}$ fatigued patient might ambulate less and therefore be less likely to experience a fall. Also, it should be noted that high BUN and creatinine levels can be indicative of conditions other than volume depletion, like a hypercatabolic state. In addition to potentially indicating volume depletion, electrolyte abnormalities like low sodium can occur secondary to other conditions like syndrome of inappropriate antidiuretic hormone secretion (SIADH). Hyponatraemia can result in deficits including altered cognitive status and weakness. ${ }^{1036}$ Lacking significant positive associations between other volume depletion-related laboratory values, it is likely that the relationship between hyponatraemia and hospital-acquired falls is related to deficits arising from hyponatraemia rather than directly related to volume depletion. In addition, we observed that the odds of experiencing a hospital-acquired fall appear to increase as sodium levels decrease independent

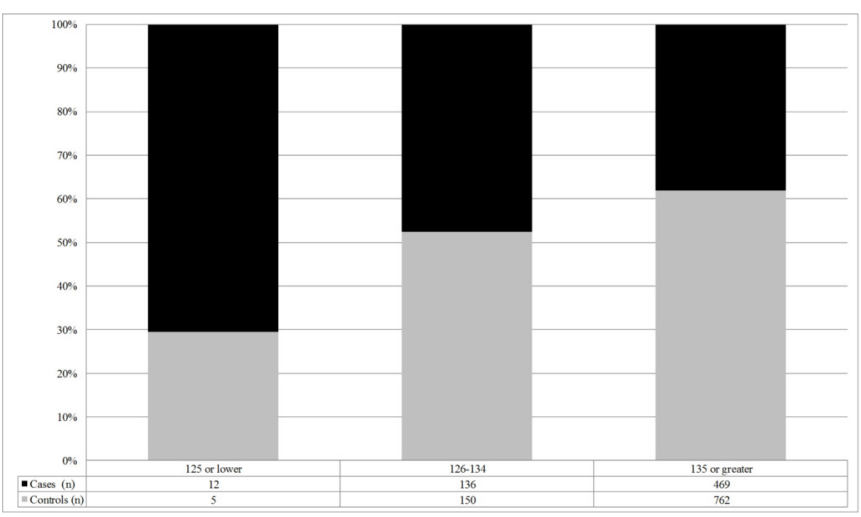

Figure 1 Proportions of cases and controls among serum sodium levels. This figure displays three categories of serum sodium levels including $125 \mathrm{mEq} / \mathrm{L}$ or lower, 126 to $134 \mathrm{mEq} / \mathrm{L}$ and $135 \mathrm{mEq} / \mathrm{L}$ or greater. Of those cases and controls with these three different serum sodium levels, the black bars indicate the proportion that are cases and the grey bars indicate the proportion that are controls. Included at the bottom of the figure is the number of cases and controls within each category of serum sodium level. of high BUN, high creatinine, a high BUN to creatinine ratio and other risk factors. This pattern further suggests that hyponatraemia is a risk factor for experiencing a hospital-acquired fall and that volume depletion does not appear to be the casual pathway.

Our findings add to previous work examining the relationship between hyponatraemia and hospital-acquired falls. An observational study conducted in a single hospital in Japan with a sample containing 97 fallers found that when controlling for age, comorbidities and increases in sedative doses, hyponatraemia (serum sodium $<135 \mathrm{mEq} / \mathrm{L}$ ) significantly increased the odds of experiencing a hospital-acquired fall $(\mathrm{OR}=1.751){ }^{25}$ In addition, an observational study examining hyponatraemia and hospital-acquired falls among a psychiatric population found that when controlling for age, antiepileptic drug use and selective serotonin reuptake inhibitor use, hyponatraemia significantly increased the odds of experiencing a hospital-acquired fall $(\mathrm{OR}=4.38){ }^{24}$ In contrast, a case-control study found that low sodium was not significantly associated with experiencing a hospital-acquired fall in a population of only those aged 65 years and older. ${ }^{23}$ However, this study was limited by sample size with only 62 fallers and 62 controls which may have limited the statistical power of its findings. Collectively, it is not easy to determine the relative contribution of our work because not all of these studies provided an operational definition of hyponatraemia.

The relationship between hyponatraemia and non-hospital acquired falls has also been examined in an observational study of fall-related and non-fall related geriatric trauma admissions. The investigators of that study determined that when controlling for potential confounders such as age and pre-existing conditions, patients with fall-related admissions were significantly more likely to have low sodium levels $(\mathrm{OR}=1.81) .{ }^{41}$ Also, investigators using a case-control study design, including patients admitted to the emergency room with and without chronic hyponatraemia, found that patients with chronic hyponatraemia were significantly more likely to have experienced a fall after controlling for covariates $(\mathrm{OR}=67) .{ }^{42}$ In a similar case-control study including geriatric patients that were admitted with and without hyponatraemia, investigators found that when controlling for covariates such as age, gender, admitting diagnosis and medications, patients with hyponatraemia were significantly more likely to have a fall associated with their admission (eg, as a presenting complaint) $(\mathrm{OR}=3.12) .{ }^{43}$ In addition, 
investigators examining hyponatraemia among community-dwelling older adults found that after adjusting for age, gender and diuretic use, persons with hyponatraemia were significantly more likely to have experienced a fall $(\mathrm{p}=0.01) .{ }^{44}$

Unlike most previous work related to laboratory values and falls, our study is informed by a conceptual framework that hypothesised the relationship between laboratory values and fall risk. ${ }^{38}$ Additional strengths of this study include the matching strategy and large sample size. Matching was based on nursing unit, date and time of fall, and length of stay which should control for environmental and staffing factors at the time of the fall. In previous research, measures of the hospital environment such as physical measures (eg, unit layout), resource availability measures (eg, nurse staffing) and culture measures (eg, magnet or teaching status) have been significantly associated with patient outcomes. ${ }^{45-47}$ In addition, at the unit environment level, researchers have found that some medical units tend to have persistently low fall rates, whereas other medical units have persistently high fall rates. ${ }^{47}$ The environment appears to have an effect on patient falls and it is, therefore, important to account for this variation. However, it should also be noted that our design cannot control for all environmental factors like location of patient room relative to the nursing station.

A limitation of this study is that only laboratory values were used as potential indicators of volume depletion. Further, the abnormal laboratory values of low sodium, high BUN, high creatinine and a high BUN to creatinine ratio are not specific to volume depletion but can also potentially indicate conditions other than volume depletion. For instance, hyponatraemia can occur as a result of SIADH. In addition, elevated BUN, creatinine and BUN to creatinine ratio levels can be seen in conditions such as CHF, sepsis, gastrointestinal obstruction and internal bleeding. ${ }^{35}$ Although these abnormal laboratory values are not specific to volume depletion, they do have clinical value in determining whether a patient is volume depleted. For instance, increases in blood urea levels have been significantly associated with decreased hydration status. ${ }^{34}$ In addition, if volume depletion is caused by vomiting, diarrhoea, diuretics or adrenal insufficiency, then the patient is likely to also be hyponatraemic. ${ }^{29}$ No laboratory value gold standard exists for identifying volume depletion; however, the laboratory values used in this study are commonly used in clinical practice and have been used in prior research as markers for volume depletion..$^{30-33}{ }^{35}$ Future work to further examine the potential relationship between volume depletion and hospital-acquired falls should consider using other potential indicators such as urine output, urine-specific gravity or orthostatic blood pressure. However, laboratory values are frequently collected in the hospital setting and are relatively reliable measures that have been neglected in previous falls research.

Additional limitations include that this was a secondary data analysis. This limited us to the exposure data available in the existing data set. Also, this study is limited by selecting for only those hospitalised patients who received laboratory results within their last 24 hours of hospitalisation prior to the fall index time. This, as well as sampling from one area of the country, limits the generalisability of these findings. However, the sample for this study was collected from four hospitals which contributes to the generalisability of these findings. Additional limitations include that observational research is susceptible to threats to internal validity including the incomplete control of potential confounders. However, we believe that our matching strategy helped to control for the relevant potential confounders of length of stay, nurse staffing, unit culture and unit environment.

\section{CONCLUSION}

In this matched case-control study, we found a strong relationship between hyponatraemia and fall risk in hospitalised patients. This relationship is independent of increased BUN, creatinine and BUN to creatinine ratio as well as independent of demographic risk factors and other patientlevel risk factors for hospital falls. Conversely, we found no other associations of laboratory findings consistent with volume depletion and increased risk of falls. It is possible that other indicators of volume depletion, like orthostatic hypotension, are necessary for the examination of this relationship. The results of this study do indicate that abnormal laboratory values, like low sodium, can be useful for identifying hospitalised patients at increased risk of experiencing a fall. Symptoms associated with hyponatraemia, including mental status changes, can be addressed with system-level and patient-level interventions like modifying the patient environment and regular patient surveillance. Further investigation into abnormal laboratory values as predictors of hospitalised-acquired falls is warranted, and if validated, should be added to currently used fall risk scales.

\section{Author affiliations}

${ }^{1}$ Departments of Biobehavioral Nursing and Family, Community, and Health System Science, University of Florida College of Nursing, Gainesville, Florida, USA

${ }^{2}$ Clinical and Translational Science Institute, University of Florida, Gainesville, Florida, USA

${ }^{3}$ Division of Research on Healthcare Value, Equity, and the Lifespan, RTI International, Research Triangle Park, NC, USA

${ }^{4}$ Center for Innovation on Disability and Rehabilitation Research (CINDRR), Malcom Randall VAMC, Gainesville, Florida, USA

${ }^{5}$ Methodist Healthcare University Hospital, Memphis, Tennessee, USA

${ }^{6}$ Department of Preventive Medicine, University of Tennessee Health Science Center, Memphis, Tennessee, USA

${ }^{7}$ Center of Excellence in Critical and Complex Care, The Ohio State University College of Nursing, Columbus, Ohio, USA

${ }^{8}$ Geriatric Research Education and Clinical Centers (GRECC), Malcom Randall VAMC, Gainesville, Florida, USA

${ }^{9}$ Department of Epidemiology, University of Florida, Gainesville, Florida, USA

Acknowledgements Publication of this article was funded in part by the University of Florida Open Access Publishing Fund.

Contributors All authors were involved in formulating the study concept/design. AMC, PAR and RIS were involved in the acquisition of the data and EAF, MTW and RIS performed the statistical analysis. EAF, RJL, MTW, AMMD, LCM and RIS were involved in the interpretation of the data, and all of the authors participated in the preparation of this manuscript. In addition, all authors approved the submitted manuscript. 
Funding This work was supported by the National Institute on Aging (R01AG033005 to RIS) and the National Center for Advancing Translational Sciences of the National Institutes of Health under University of Florida Clinical and Translational Science Awards (TL1TR001428 andUL1TR001427 to EAF).

Disclaimer The content is solely the responsibility of the authors and does not necessarily represents the official views of the National Institutes of Health.

Ethics approval Data collection was approved by the Institutional Review Board of the University of Tennessee Health Science Center. The secondary analysis was approved by the University of Florida Institutional Review Board.

Provenance and peer review Not commissioned; externally peer reviewed.

Data sharing statement № additional data are available for sharing.

Open Access This is an Open Access article distributed in accordance with the terms of the Creative Commons Attribution (CC BY 4.0) license, which permits others to distribute, remix, adapt and build upon this work, for commercial use, provided the original work is properly cited. See: http://creativecommons.org/ licenses/by/4.0/

(C) Article author(s) (or their employer(s) unless otherwise stated in the text of the article) 2017. All rights reserved. No commercial use is permitted unless otherwise expressly granted.

\section{REFERENCES}

1. Centers for Disease Control and Prevention. Falls among older adults: an overview. https://www.cdc.gov/ HomeandRecreationalSafety/Falls/adultfalls.html. (accessed Jun 2015).

2. Ganz DA, Huang C, Saliba D, et al. Agency for Healthcare Research and Quality. Preventing falls in hospitals: a toolkit for improving quality of care. 2013. https://www.ahrq.gov/sites/default/files/ publications/files/fallpxtoolkit.pdf. (accessed Jun 2015).

3. Angalakuditi M, Coley KC, Kirisci L, et al. A case-control study to assess the impact of anemia and other risk factors for in-hospital falls. J Patient Saf 2007;3:16-21.

4. Hendrich A, Nyhuis A, Kippenbrock T, et al. Hospital falls: development of a predictive model for clinical practice. Appl Nurs Res 1995;8:129-39.

5. Hendrich AL, Bender PS, Nyhuis A. Validation of the Hendrich II Fall Risk Model: a large concurrent case/control study of hospitalized patients. Appl Nurs Res 2003;16:9-21.

6. Krauss MJ, Evanoff B, Hitcho E, et al. A case-control study of patient, medication, and care-related risk factors for inpatient falls. $J$ Gen Intern Med 2005;20:116-22.

7. Yauk S, Hopkins BA, Phillips CD, et al. Predicting in-hospital falls: development of the Scott and White Falls Risk Screener. J Nurs Care Qual 2005;20:128-33.

8. Morse JM, Tylko SJ, Dixon HA. Characteristics of the fall-prone patient. Gerontologist 1987;27:516-22.

9. Ash KL, MacLeod P, Clark L. A case control study of falls in the hospital setting. J Gerontol Nurs 1998;24:7-9.

10. Rao SS. Prevention of falls in older patients. Am Fam Physician 2005;72:81-8.

11. Morse JM, Morse RM, Tylko SJ. Development of a scale to identify the fall-prone patient. Can J Aging 1989;8:366-77.

12. Oliver D, Britton M, Seed P, et al. Development and evaluation of evidence based risk assessment tool (STRATIFY) to predict which elderly inpatients will fall: case-control and cohort studies. BMJ 1997;315:1049-53.

13. Aranda-Gallardo M, Morales-Asencio JM, Canca-Sanchez JC, et al. Instruments for assessing the risk of falls in acute hospitalized patients: a systematic review and meta-analysis. BMC Health Serv Res 2013;13:122.

14. Chen YC, Chien SF, Chen LK. Risk factors associated with falls among Chinese hospital inpatients in Taiwan. Arch Gerontol Geriatr 2009:48:132-6.

15. Plati C, Lanara V, Mantas J. Risk factors responsible for patients falls. Scand J Caring Sci 1992;6:113-8

16. Dharmarajan TS, Avula S, Norkus EP. Anemia increases risk for falls in hospitalized older adults: an evaluation of falls in 362 hospitalized, ambulatory, long-term care, and community patients. J Am Med Dir Assoc 2006; 7:287-93.

17. O'Hagan $\mathrm{C}, \mathrm{O}^{\prime}$ Connell $\mathrm{B}$. The relationship between patient blood pathology values and patient falls in an acute-care setting: a retrospective analysis. Int J Nurs Pract 2005;11:161-8.
18. Church S, Robinson TN, Angles EM, et al. Postoperative falls in the acute hospital setting: characteristics, risk factors, and outcomes in males. Am J Surg 2011;201:197-202.

19. Capone LJ, Albert NM, Bena JF, et al. Predictors of a fall event in hospitalized patients with cancer. Oncol Nurs Forum 2012;39:E407-E415.

20. Byers V, Arrington ME, Finstuen K. Predictive risk factors associated with stroke patient falls in acute care settings. J Neurosci Nurs 1990;22:147-54.

21. Stenvall M, Olofsson $B$, Lundström $M$, et al. Inpatient falls and injuries in older patients treated for femoral neck fracture. Arch Gerontol Geriatr 2006;43:389-99.

22. Chu LW, Pei CK, Chiu A, et al. Risk factors for falls in hospitalized older medical patients. J Gerontol A Biol Sci Med Sci 1999;54:M38-M43.

23. Walker PC, Alrawi A, Mitchell JF, et al. Medication use as a risk factor for falls among hospitalized elderly patients. Am J Health Syst Pharm 2005;62:2495-9.

24. Bun S, Serby MJ, Friedmann P. Psychotropic medication use, hyponatremia, and falls in an inpatient population: a retrospective study. J Clin Psychopharmacol 2011;31:395-7.

25. Tachi T, Yokoi T, Goto C, et al. Hyponatremia and hypokalemia as risk factors for falls. Eur J Clin Nutr 2015;69:205-10.

26. Robinson SB, Rosher RB. Can a beverage cart help improve hydration? Geriatr Nurs 2002;23:208-11.

27. Rubenstein LZ. Falls in older people: epidemiology, risk factors and strategies for prevention. Age Ageing 2006;35:ii37-ii41.

28. Ungar A, Rafanelli M, lacomelli I, et al. Fall prevention in the elderly. Clin Cases Miner Bone Metab 2013;10:91-5.

29. Morley JE. Dehydration, Hypernatremia, and Hyponatremia. Clin Geriatr Med 2015;31:389-99.

30. Gross CR, Lindquist RD, Woolley AC, et al. Clinical indicators of dehydration severity in elderly patients. J Emerg Med 1992;10:267-74.

31. McGee S. Is this patient hypovolemic? JAMA 1999;281:1022-9.

32. Sinert R, Spektor M. Clinical assessment of hypovolemia. Ann Emerg Med 2005;45:327-9.

33. Riccardi A, Chiarbonello B, Minuto P, et al. Identification of the hydration state in emergency patients: correlation between caval index and BUN/creatinine ratio. Eur Rev Med Pharmacol Sci 2013;17:1800-3.

34. Shepherd J, Hatfield S, Kilpatrick ES. Is there still a role for measuring serum urea in an age of eGFR? evidence of its use when assessing patient hydration. Nephron Clin Pract 2009;113:c20 3-c206.

35. Robinson $\mathrm{BE}$, Weber $\mathrm{H}$. Dehydration despite drinking: beyond the BUN/creatinine ratio. J Am Med Dir Assoc 2004; 5:S67-71.

36. Van Leeuwen AM, Bladh ML. Davis's comprehensive handbook of laboratory \& diagnostic tests with nursing implications. 6th ed. Philadelphia, PA: F.A. DavisCo, 2015.

37. Simon EE. Hyponatremia evaluation and treatment. New York, NY: Springer, 2013.

38. Choi YS, Lawler E, Boenecke CA, et al. Developing a multi-systemic fall prevention model, incorporating the physical environment, the care process and technology: a systematic review. $J$ Adv Nurs 2011;67:2501-24.

39. SAS Institute Inc. SAS/STAT 9.2 user's guide conditional logisitc regression for matched pairs data. 2 nd ed. Cary, NC: SAS Institute Inc, 2009.

40. Allison PD. Logistic regression using SAS: theory and application. 2nd ed. Cary, NC: SAS Institute Inc, 2012. https://support.sas.com/ documentation/cdl/en/statug/63033/HTML/default/viewer.htm\# statug_logistic_sect062.htm. (accessed Aug 2016).

41. Rittenhouse KJ, To T, Rogers A, et al. Hyponatremia as a fall predictor in a geriatric trauma population. Injury 2015; 46:119-23.

42. Renneboog B, Musch W, Vandemergel X, et al. Mild chronic hyponatremia is associated with falls, unsteadiness, and attention deficits. Am J Med 2006;119:71.e1-71.e8.

43. Ahamed S, Anpalahan M, Savvas S, et al. Hyponatraemia in older medical patients: implications for falls and adverse outcomes of hospitalisation. Intern Med J 2014;44:991-7.

44. Gunathilake R, Oldmeadow C, McEvoy M, et al. Mild hyponatremia is associated with impaired cognition and falls in communitydwelling older persons. J Am Geriatr Soc 2013; 61:1838-9.

45. Lake ET, Shang J, Klaus S, et al. Patient falls: association with hospital magnet status and nursing unit staffing. Res Nurs Health 2010;33:413-25. 
46. Everhart D, Schumacher JR, Duncan RP, et al. Determinants of hospital fall rate trajectory groups: a longitudinal assessment of nurse staffing and organizational characteristics. Health Care Manage Rev 2014;39:352-60.
47. Staggs VS, Mion LC, Shorr RI. Consistent differences in medical unit fall rates: implications for research and practice. J Am Geriatr Soc 2015;63:983-7. 